\title{
Synovial pH Is As Specific As Synovial Leukocyte Count But Less Sensitive For The Diagnosis of Chronic Prosthetic Joint Infection
}

Thomas Ackmann ( $\square$ thomas.ackmann@ukmuenster.de)

Muenster University Hospital

Jan Schwarze

Muenster University Hospital

Georg Goshger

Muenster University Hospital

Tom Schmidt-Braekling

Muenster University Hospital

Sebastian Klingebiel

Muenster University Hospital

Burkhard Moellenbeck

Muenster University Hospital

Christoph Theil

Muenster University Hospital

\section{Research Article}

Keywords: periprosthetic joint infection, synovial fluid, $\mathrm{pH}$

Posted Date: May 6th, 2021

DOl: https://doi.org/10.21203/rs.3.rs-465751/v1

License: (c) (i) This work is licensed under a Creative Commons Attribution 4.0 International License. Read Full License 


\section{Abstract}

Synovial fluid markers have been shown to be most accurate in diagnosing prosthetic joint infection (PJI). An inverse correlation for synovial leukocyte count and synovial $\mathrm{pH}$ is known assuming that leukocyte metabolism causes synovial fluid acidosis. This study analysis synovial $\mathrm{pH}$ as a potential marker for PJI. We prospectively included 92 patients who presented with painful total joint arthroplasty (TJA) of the hip (THA; $n=25)$ or knee (TKA, $n=67)$. Joints were diagnosed as infected $(n=30)$ or aseptic $(n=62)$ based on the Musculoskeletal Infection Society criteria of 2018. An ideal cut-off value for synovial $\mathrm{pH}$ and the sensitivity and specificity were calculated. Additionally, the sensitivity and specificity were calculated for synovial white blood cell (WBC) count (cut-off $>3000$ leukocytes) and percentage of neutrophils (PMN\%, cut-off $>80 \%$ ). The median synovial $\mathrm{pH}$ level was significantly lower in the group with chronic PJI (7.09 vs. $7.27 ; p<0.001)$ compared to implants with aseptic failure. The calculated optimal cut-off value was 7.11 (AUC 0.771 ) with a sensitivity of $53 \%$ and specificity of $89 \%$. However, the sensitivity and specificity of synovial WBC count were $90 \%$ and $88 \%$ and for synovial PMN\% $73 \%$ and $98 \%$, respectively. Synovial pH may be a cost-effective parameter to diagnose chronic PJI.

\section{Introduction}

Periprosthetic joint infection (PJI) is one of the most feared complications that can occur after total joint arthroplasty (TJA) of the hip (THA) or knee (TKA) [1, 2]. Its incidence after primary TJA around 1-2\% [3], but due to the increasing number of performed joint replacements, the number of revision arthroplasties due to PJI will continue to rise in the coming years [4-6]. Timely and accurately diagnosing of PJI especially in low grade cases - is still challenging in daily clinical practice and since to date no optimal, universal test is available $[6,7]$.

Currently, the diagnosis of PJI is usually established using the criteria published by the Musculoskeletal Infection Society (MSIS) and by the International Consensus Meeting (ICM) proceedings $[8,9]$. Beside the clinical findings as well as serum and microbiological testing, both definitions include synovial white blood cell (WBC) count and percentage of synovial polymorphonucleocytes (PMN\%). Synovial fluid analysis appears to be most reliable at the moment $[10,11]$ but it still has no perfect diagnostic accurary and the optimal threshold for different joints and organisms are debated $[9,12,13]$.

The potential role of synovial fluid $\mathrm{pH}$ as a biomarker to differentiate identify joint with inflammatory joint disease and those with normal joints was investigated $(n=16)$ as early as 1966 [14]. Generally, the pH is a scale used to specify the acidity or basicity of an aqueous solution[15] and acidic solutions (solutions with higher concentrations of $\mathrm{H}^{+}$ions) are measured to have lower $\mathrm{pH}$ values than alkaline solutions. $\mathrm{A}$ previous study on synovial fluid $\mathrm{pH}$ in native joints[14] showed a significantly lower synovial $\mathrm{pH}$ value in cases of inflammatory joint disease than in non-inflammatory joints (7.223 vs. 7.434$)$. Later research $(n=$ 22) found a close correlation for an increasing synovial WBC count and an associating decreasing synovial pH in septic native joint arthritis [16]. The demonstrated inverse correlation of WBCs and pH supports the suggestion that white blood cell metabolism is responsible for synovial fluid acidosis. 
Considering that PJI is associated with an increased synovial WBC count $[8,9]$, this study evaluates synovial $\mathrm{pH}$ in patients undergoing revision arthroplasty of the hip or knee as a potential synovial biomarker for chronic PJI and compares its accuracy with synovial WBC count and PMN\%.

\section{Methods}

Prior to this prospective investigation approval of the institutional review board of the authors' institution was obtained (ethics committee of the University of Muenster, ref. no. 2019-666-f-S) and it was registered in the German Clinical Trials Register (Registration number: DRKS00021038; date of registration: 18/03/2020). Written consent was obtained from all participants and the study was conducted according to the principles of the World Medical Association Declaration of Helsinki. No specific source of funding was required for this study.

All patients who presented to our tertiary centre for revision arthroplasty with a potential indication for revision either for chronic PJI or for aseptic causes routinely undergo tests of serum, synovial and microbiological parameters prior to rule out PJI. For this study we added a synovial pH-test to the diagnostic preoperative algorithm and analyse the preliminary findings of data collected between August 2019 and October 2020. Synovial fluid was collected in safePICO syringes (Radiometer GmbH, Krefeld, Germany) with a built-in mixing ball and dry electrolyte-balanced heparin, helping to minimize the risk for clot formation and measured by potentiometry on a commercial blood gas analyser (ABL 90 FLEX PLUS, Radiometer $\mathrm{GmbH}$, Krefeld, Germany).

In our practice all patients undergo analysis of serum c-reactive protein (CRP), serum interleukin-6 (II-6), serum and synovial WBC count, differentiation of synovial WBCs including PMN\% and synovial microbiology culture. Patients who had surgery within the last 4 weeks, those with chronic systemic inflammation such as rheumatoid arthritis, those with confirmed inflammatory diseases of other organs such as pneumonia and urinary tract infection, those with malignancies were not screened using synovial $\mathrm{pH}$ testing. For patients that underwent revision arthroplasty $(n=62)$, either for chronic PJI $(n=30)$ or aseptic failure $(n=32)$, a minimum of five intraoperative tissue samples for microbiology cultures were taken additionally to the synovial fluid, and another tissue sample was taken for histological again applying the criteria by the MSIS. All taken tissue and synovial fluid samples were cultured for a minimum of 14 days on Columbia blood agar, chocolate agar and Schaedler agar for microbiological testing. Furthermore, 34 patients were evaluated preoperatively, but did not undergo surgery so far because they declined surgery at this point or a non-operative management has been recommended, resulting in a total of 96 patients who were eligible for analysis. Participants meeting the study's inclusion criteria were prospectively evaluated and classified as infected $(n=30)$, not-infected $(n=62)$ or inconclusive $(n=4)$ as defined in the ICM proceedings 2018 . Those with an inconclusive score were excluded from the statistical analysis. In total 92 patients (25 THA, 67 TKA, 46 male, median age 69 (IQR $60-76)$ were included in this study. Thirty patients (19 TKA, 11 THA) were assigned to the PJI group, while the remaining 62 patients were not considered infected (48 TKA, 14 THA). 


\section{Statistical analysis}

Excel (Microsoft Corporation, Redmont, Washington, USA) and Statistical Package for the Social Sciences Statistics for Windows version 25 (IBM Corporation, Armonk, NY, USA) were used for statistical analysis of pseudonymized patient data. The Shapiro-Wilk test and descriptive statistics were used to analyse distribution of data. For parametric data the means and ranges were calculated; the medians and 25-75\% interquartile ranges (IQRs) were obtained for non-parametric data. The non-parametric analyses were performed using the Mann-Whitney $U$ test and frequencies were given for categorical variables that were compared in contingency tables using the chi-squared test.

Receiver operating characteristics (ROC) analyses with presentation of the area under the curve (AUC) with $95 \%$ confidence interval $(\mathrm{Cl})$ were used for statistical evaluation. To determine the optimal cut-off value for synovial $\mathrm{pH}$ the Youden's index was used. Based on the determined cut-off value for synovial $\mathrm{pH}$ and the given cut-off values for WBC count $(>3000 / \mu \mathrm{l})$ and PMN\% $(>80 \%)[8,9]$, sensitivities and specificities were calculated for each biomarker from contingency tables. Statistical significance was set at $p \leq 0.05$.

\section{Results}

The median synovial pH level was significantly lower in the patients diagnosed with chronic PJI (7.09 $(I Q R, 6.98-7.26))$ compared to the patients with aseptic failure (7.27 (IQR, 7.17-7.32) $(p<0.001)$.

The ROC curve analysis revealed the lowest AUC for synovial pH, at 0.771 (95\% confidence interval (95\% $\mathrm{Cl}), 0.664-0.878)$. Using Youden's index, the optimal cut-off value was $7.11 \mathrm{for}$ synovial $\mathrm{pH}$ discriminating between chronic PJI and aseptic failure. Fifty-five of 62 not infected samples had a synovial pH value below 7.11 and 16 of 30 infected samples had a synovial pH above 7.11. Applying this threshold, a specificity of $89 \%$ and sensitivity of $53 \%$ were calculated. The positive predictive value to correctly identify chronic PJI was $69 \%$ and the negative predictive value was $79 \%$ (Table 1).

Table 1

Results for synovial pH value, synovial white blood cell (WBC) count and percentage of synovial neutrophils (PMN\%) in diagnosing prosthetic joint infection (PJI).

\begin{tabular}{|llllll|}
\hline Markers & Used cut-off values & Sensitivity (\%) & Specificity (\%) & PPV & NPV \\
\hline pH & $<7.11$ & 53 & 89 & 0.6956 & 0.7971 \\
\hline WBC count & $>3000 / \mu \mathrm{l}$ & 90 & 88 & 0.7941 & 0.9464 \\
\hline PMN\% & $>80 \%$ & 73 & 98 & 0.8529 & 0.8787 \\
\hline
\end{tabular}

In comparison, the median level of synovial WBC count (14905 (IQR, 6692-28898) vs. 660 (IQR, 3101422), $\mathrm{p}<0.001)$ and synovial PMN\% (90.6\% (IQR, 76.6-94.6\%) vs. $26.9 \%($ IQR, 19.5-45.3\%), p < 0.001) were significantly higher in patients with chronic PJI compared to patients with aseptic failure. 
The highest AUC was shown for synovial PMN\% with 0.962 (95\% Cl, 0.911-1.000), followed by synovial WBC count with $0.936(95 \% \mathrm{Cl}, 0.880-0.993)$. Using the given cut-off values, the sensitivity and specificity of synovial WBC count were $90 \%$ and $88 \%$ and $73 \%$ and $98 \%$ for synovial PMN\%, respectively.

In 25 of 30 cases (83.3\%) with chronic PJI a causative bacterium was identified (Table 2), while five (16.7\%) patients had culture-negative infection and four (13.3\%) presented with a polymicrobial infection. In case of the five culture-negative patients one patient had a fistula and the other cases each had an elevated CRP, elevated synovial WBC count and elevated synovial PMN\%. There were no unexpected positive cultures in the group that was considered not infected preoperative.

Table 2

List of the identified bacteria for the 25 patients the PJI group.

\begin{tabular}{|ll|}
\hline Culture organism & Frequency [\%] \\
\hline Staphylococcus lugdunensis & 3.8 \\
\hline Enterobacter cloacae complex & 5.8 \\
\hline Corynebacterium & 3.8 \\
\hline Staphylococcus epidermidis & 17.2 \\
\hline Escherichia coli & 3.8 \\
\hline Streptococcus agalactiae & 3.8 \\
\hline Enterococcus faecalis & 7.7 \\
\hline Staphylococcus caprae & 3.8 \\
\hline Staphylococcus aureus & 3.8 \\
\hline Staphylococcus capitis & 7.7 \\
\hline Streptococcus dysgalactiae & 3.8 \\
\hline
\end{tabular}

\section{Discussion}

PJI continues to affect a remarkable number of patients who undergo TJA but the accurate diagnosis is still challenging in daily clinical practise and the search for potential serological and synovial biomarkers is ongoing [17-19]. This study evaluates synovial $\mathrm{pH}$ as a potential novel marker for chronic PJI. Using the calculated cut-off value of 7.11, synovial pH showed good potential to diagnose chronic PJI with a high specificity (89\%), however it had a poor sensitivity (53\%). Despite the low sensitivity, we were able to show a significant difference for the $\mathrm{pH}$ value of the chronic septic and aseptic cases after TJA of the knee or hip. 
A high synovial WBC and the leukocytes metabolism is responsible for decreasing synovial pH [8]. Furthermore, the presence of bacteria leads to high synovial lactate concentrations and this correlates with a drop of synovial $\mathrm{pH}$ [20]. For the present cohort culture negative infection was rare and four patients had polymicrobial infection and in general 11 different organisms were detectable which poses the question if and to what extent [20], different bacteria result in varying synovial $\mathrm{pH}$ value as it has been reported for native joint infections [20]. Furthermore, previous studies have discussed and proposed different thresholds for leukocyte count and differential depending on the type of infection (low-grade or high-grade) or even individual species. This may also be the case for synovial $\mathrm{pH}$ and should be answered in further investigations with larger numbers.

In addition to the other possible influencing factors such as gender, age, affected joint[21] or comorbidities, the size of the implanted endoprosthesis can be of relevance and needs further investigations. Milošev et al.[21] observed a small but statistically significant difference in the $\mathrm{pH}$ of synovial fluid between natural joints with degenerative diseases and joints with metal implants. It has been hypothesized that the release for metal ions from joint implants may lead to a decrease in synovial $\mathrm{pH}$. Therefore it is possible that the $\mathrm{pH}$ may be influenced by implant size with megaprosthesis potentially leading to a lower synovial $\mathrm{pH}$ and potentially lower diagnostic thershold. Furthermore, a longlasting TJA may have released more metal ions over time due to mechanical wear which might also lower the synovial pH. However, the usefulness of synovial cell count and differential has been generally questioned in this context when a form of metallosis or adverse local tissue reactions is present. Nonetheless, to our knowledge the effect of implant size on synovial leukocyte count and differentiation has not been explored to the author's knowledge although a difference is possible. Therefore, future studies should also investigate synovial pH if increased metal ion release is present (metal-on-metal bearing, metallosis, megaprostheses, long-lasting implants) in conjunction with synovial leukocytes.

The combination of microbiological long-term incubation, leukocyte count and leukocyte differentiation is the current gold standard analysis of synovial fluid [12]. Especially WBC count and PMN\% provide high sensitivities and specificities as demonstrated in numerous studies $[8,9,12]$. While this is the first study to investigate synovial pH for chronic PJI, synovial WBC count and PMN\% have already been examined in numerous previously studies but there is still disagreement among orthopaedic surgeons about the optimal threshold [22]. For our investigation we used the thresholds given by MSIS 2018[8] and ICM proceedings[9] to determine the sensitivity and specificity. For our cohort the specificity of WBC count (88\%) and PMN\% (98\%) was as high as for $\mathrm{pH}(89 \%)$, whereas the sensitivity was best for WBC count (90\%), but lower for PMN\% (73\%) and pH (53\%). However, other institutions[12] or authors[23] recommend lower cut-off values to detect low-grade infections due to the fact, that the change of synovial fluid parameters depends on the virulence of bacteria[12] and may be as low as 1500 leukocytes and $65 \% \mathrm{PMN}$ which must also be considered when interpreting synovial $\mathrm{pH}$ testing and the sensitivity reported here.

In conclusion, synovial pH can help to diagnose chronic PJI of lower extremity arthroplasty given its very good specificity, however in this preliminary cohort it showed poor sensitivity and therefore must be 
combined with other serum and synovial biomarkers.

\section{Declarations}

\section{Acknowledgments}

The author's acknowledge support by the Open Access Fund of the Universitiy of XXXX who paid the publication fee.

\section{Author's contributions}

TA review of literature, conception and design, acquisition of data, analysis and interpretation of data, statistical analysis, drafting of the manuscript; JS review of literature acquisition of data, analysis and interpretation of data, statistical analysis, critical revision of the manuscript; GG conception and design, critical revision of the manuscript; TSB critical revision of the manuscript, conception and design; BM and SK conception and design, critical revision of the manuscript; CT drafting of the manuscript, analysis and interpretation of data, administrative support, conception and design. All authors read and approved the final manuscript.

\section{Competing interests}

The author's declare no competing interests.

\section{References}

1. Kamath, A. F. et al. Quantifying the Burden of Revision Total Joint Arthroplasty for Periprosthetic Infection. The Journal of arthroplasty. 30, 1492-1497 (2015).

2. Nikolaus, O. B. et al. Factors Associated With 20-Year Cumulative Risk of Infection After Aseptic Index Revision Total Knee Arthroplasty. The Journal of arthroplasty. 31, 872-877 (2016).

3. Zmistowski, B., Restrepo, C., Huang, R., Hozack, W. J. \& Parvizi, J. Periprosthetic joint infection diagnosis: a complete understanding of white blood cell count and differential. The Journal of arthroplasty. 27, 1589-1593 (2012).

4. Inacio, M. C. S., Paxton, E. W., Graves, S. E., Namba, R. S. \& Nemes, S. Projected increase in total knee arthroplasty in the United States - an alternative projection model. Osteoarthritis and cartilage. 25, 1797-1803 (2017).

5. Kim, S. H., Gaiser, S. \& Meehan, J. P. Epidemiology of primary hip and knee arthroplasties in Germany: 2004 to 2008. The Journal of arthroplasty. 27, 1777-1782 (2012).

6. Zimmerli, W., Trampuz, A. \& Ochsner, P. E. Prosthetic-joint infections. The New England journal of medicine. 351, 1645-1654 (2004).

7. Shahi, A. \& Parvizi, J. The role of biomarkers in the diagnosis of periprosthetic joint infection. EFORT open reviews. 1, 275-278 (2016). 
8. Parvizi, J. et al. The 2018 Definition of Periprosthetic Hip and Knee Infection: An Evidence-Based and Validated Criteria. The Journal of arthroplasty. 33, 1309-13142 (2018).

9. Shohat, N. et al. Hip and Knee, What is the Definition of a Periprosthetic Joint Infection (PJI) of the Knee and the Hip? Can the Same Criteria be Used for Both Joints?: Proceedings of International Consensus on Orthopedic Infections. The Journal of arthroplasty 34, S325-S327(2019).

10. Sousa, R., Serrano, P., Gomes Dias, J., Oliveira, J. C. \& Oliveira, A. Improving the accuracy of synovial fluid analysis in the diagnosis of prosthetic joint infection with simple and inexpensive biomarkers: C-reactive protein and adenosine deaminase. The bone \& joint journal. 99-B, 351-357 (2017).

11. Lee, Y. S. et al. Synovial Fluid Biomarkers for the Diagnosis of Periprosthetic Joint Infection: A Systematic Review and Meta-Analysis. The Journal of bone and joint surgery. American volume. 99, 2077-2084 (2017).

12. Mühlhofer, H. et al. Diagnostik der periprothetischen Infektion: Entwicklung eines evidenzbasierten Algorithmus der Arbeitsgruppe implantatassoziierte Infektion der Arbeitsgemeinschaft Endoprothetik. Der Orthopade. https://doi.org/10.1007/s00132-020-03940-6 (2020).

13. Osmon, D. R. et al. Diagnosis and management of prosthetic joint infection: clinical practice guidelines by the Infectious Diseases Society of America. Clinical infectious diseases: an official publication of the Infectious Diseases Society of America. 56, e1-e25 (2013).

14. Cummings, N. A. \& Nordby, G. L. Measurement of synovial fluid pH in normal and arthritic knees. Arthritis and rheumatism. 9, 47-56 (1966).

15. Covington, A. K., Bates, R. G. \& Durst, R. A. Definition of pH scales, standard reference values, measurement of $\mathrm{pH}$ and related terminology (Recommendations 1984). Pure and Applied Chemistry. 57, 531-542 (1985).

16. Ward, T. T. \& Steigbigel, R. T. Acidosis of synovial fluid correlates with synovial fluid leukocytosis. The American Journal of Medicine. 64, 933-936 (1978).

17. Ackmann, T. et al. Comparing the Diagnostic Value of Serum D-Dimer to CRP and IL-6 in the Diagnosis of Chronic Prosthetic Joint Infection. Journal of clinical medicine9 (2020).

18. Goswami, K. \& Parvizi, J. \& Maxwell Courtney, P. Current Recommendations for the Diagnosis of Acute and Chronic PJI for Hip and Knee-Cell Counts, Alpha-Defensin, Leukocyte Esterase, Nextgeneration Sequencing. Current reviews in musculoskeletal medicine. 11, 428-438 (2018).

19. Xie, K., Dai, K., Qu, X. \& Yan, M. Serum and Synovial Fluid Interleukin-6 for the Diagnosis of Periprosthetic Joint Infection. Scientific reports. 7, 1496 (2017).

20. Gobelet, C. \& Gerster, J. C. Synovial fluid lactate levels in septic and non-septic arthritides. Annals of the rheumatic diseases. 43, 742-745 (1984).

21. Milošev, I., Levašič, V., Vidmar, J., Kovač, S. \& Trebše, R. pH and metal concentration of synovial fluid of osteoarthritic joints and joints with metal replacements. Journal of biomedical materials research. Part B, Applied biomaterials. 105, 2507-2515 (2017).

22. Zahar, A. et al. How Reliable Is the Cell Count Analysis in the Diagnosis of Prosthetic Joint Infection? The Journal of arthroplasty. 33, 3257-3262 (2018). 
23. Mihalič, R., Zdovc, J., Brumat, P. \& Trebše, R. Synovial fluid interleukin-6 is not superior to cell count and differential in the detection of periprosthetic joint infection. Bone \& joint open. 1, 737-742 (2020). 